\title{
The effect of a multispecies probiotic on the composition of the faecal microbiota and bowel habits in chronic obstructive pulmonary disease patients treated with antibiotics
}

\author{
Catherina J. M. Koning ${ }^{1}$, Daisy Jonkers ${ }^{1,2}$, Hauke Smidt ${ }^{3}$, Frans Rombouts ${ }^{4,5}$, Herman-Jan Pennings ${ }^{6}$, \\ Emiel Wouters ${ }^{6,7}$, Ellen Stobberingh ${ }^{2}$ and Reinhold Stockbrügger ${ }^{1}$ \\ ${ }^{1}$ Division of Gastroenterology-Hepatology, Research Institute NUTRIM, Maastricht University Medical Centre (MUMC), \\ Maastricht, The Netherlands \\ ${ }^{2}$ Division of Medical Microbiology, Research Institute NUTRIM, MUMC, University Hospital Maastricht, PO Box 5800,6202 AZ \\ Maastricht, The Netherlands \\ ${ }^{3}$ Laboratory of Microbiology, Department of Agrotechnology and Food Sciences, Wageningen University, Wageningen, \\ The Netherlands \\ ${ }^{4}$ Laboratory of Food Microbiology, Department of Agrotechnology and Food Sciences, Wageningen University, Wageningen, \\ The Netherlands \\ ${ }^{5}$ Winclove Bio Industries B.V., Amsterdam, The Netherlands \\ ${ }^{6}$ Department of Respiratory Medicine, Centre for Integrated Rehabilitation of Organ Failure (CIRO), Horn, The Netherlands \\ ${ }^{7}$ Department of Respiratory Medicine, Research Institute NUTRIM, MUMC, Maastricht, The Netherlands
}

(Received 21 July 2009 - Revised 17 November 2009 - Accepted 18 November 2009 - First published online 21 December 2009)

\begin{abstract}
Short-term antibiotic treatment profoundly affects the intestinal microbiota, which may lead to sustained changes in microbiota composition. Probiotics may restore such a disturbance. The objective of the present study was to investigate the effect of a multispecies probiotic on the faecal microbiota during and after antibiotic intake in patients with a history of frequent antibiotic use. In this randomised, placebo-controlled, double-blind study, thirty chronic obstructive pulmonary disease (COPD) patients treated with antibiotics for a respiratory tract infection received $5 \mathrm{~g}$ of a multispecies probiotic or placebo twice daily for 2 weeks. Faecal samples were collected at $0,7,14$ and $63 \mathrm{~d}$. Changes in the composition of the dominant faecal microbiota were determined by PCR-denaturing gradient gel electrophoresis (DGGE). Changes in bacterial subgroups were determined by quantitative PCR and culture. Bowel movements were scored daily according to the Bristol stool form scale. During and after antibiotic treatment, DGGE-based similarity indices (SI) were high $(\geq 84 \%)$ and band richness was relatively low, both remaining stable over time. No difference in SI was observed between patients with and without diarrhoea-like bowel movements. The multispecies probiotic had a modest effect on the bacterial subgroups. Nevertheless, it affected neither the composition of the dominant faecal microbiota nor the occurrence of diarrhoea-like bowel movements. The dominant faecal microbiota was not affected by antibiotics in this COPD population, suggesting an existing imbalance of the microbiota, which may also have contributed to the lack of effect by probiotic intake.
\end{abstract}

Probiotics: Antibiotics: PCR-denaturing gradient gel electrophoresis: Intestinal microbiota: Bowel movements

In recent years, our knowledge on the composition of the human intestinal microbiota has greatly improved with the introduction of molecular techniques based on 16S rRNA gene sequences, confirming that the microbiota is subject specific and comprises a complex and very diverse bacterial ecosystem of which most species belong to previously unknown (i.e. uncultivated) lineages ${ }^{(1-3)}$. In healthy individuals, the dominant intestinal microbiota is found to be relatively stable over time $\mathrm{e}^{(1,4-7)}$. However, in various populations it has been demonstrated that antibiotic intake can cause a marked disturbance of the intestinal microbiota as they not only affect pathogens but also the indigenous microbiota, which may result in antibiotic-associated diarrhoea (AAD). It is widely assumed that this disturbance is short-term but recently also medium and long-term disturbances in (specific) bacterial populations have been described $^{(8-10)}$. However, there are chronically ill patients, such as patients suffering from chronic obstructive pulmonary disease (COPD), who receive frequent antibiotic therapy. COPD is characterised by a progressive airflow obstruction, associated with a chronic inflammatory response in the airways consisting mainly of neutrophils, $\mathrm{CD} 8^{+} \mathrm{T}$-cells and

Abbreviations: AAD, antibiotic-associated diarrhoea; COPD, chronic obstructive pulmonary disease; DGGE, denaturing gradient gel electrophoresis; SI, similarity indices.

* Corresponding author: Catherina J. M. Koning, fax +31 433875006, email c.koning@fdg-guest.unimaas.nl 
macrophages ${ }^{(11,12)}$. Acute exacerbations play an important role in the clinical course of the disease and are an important cause of morbidity and mortality ${ }^{(13,14)}$. Although the underlying pathogenic mechanisms are poorly understood, antibiotic therapy is frequently prescribed. The effects of such frequent antibiotic use on the intestinal microbiota are, to our knowledge, not known.

The intestinal microbiota plays an important role in human physiology, being involved in gut maturation, colonisation resistance, several metabolic processes, regulation of intestinal epithelial proliferation and modulation of the mucosal and systemic immune response ${ }^{(15-18)}$. Based on this, probiotics are often given during and after antibiotic intake and beneficial effects have been observed in the prevention and treatment of $\mathrm{AAD}^{(19-22)}$. Therefore, in the present study a multispecies probiotic was given containing nine different probiotic strains selected on the basis of their in vitro ability to inhibit growth of Clostridium spp., in vitro survival of gastrointestinal passage $^{(23)}$ (i.e. low $\mathrm{pH}(2 \cdot 5)$ as well as bile and digestive enzymes (pancreatin and pepsin)) and the absence of acquired antibiotic resistance. Moreover, a combination of strains were selected in which strain-specific properties were chosen to be additive or synergistic and mutual inhibition was absent. In addition, we previously showed that in healthy volunteers the intake of this multispecies probiotic during and after amoxycillin intake has no adverse effects and affects the faecal microbiota resulting in a faster restoration toward the pre-antibiotic state ${ }^{(10)}$.

The aim of the present study was to assess the disturbance of the dominant faecal microbiota and the possible restoration by a multispecies probiotic in patients with COPD treated with antibiotics for an acute exacerbation. Moreover, the effect on specific bacterial subgroups, using both culturedependent and molecular based techniques, bowel habits, the incidence of antibiotic resistance, endotoxins and $\mathrm{pH}$ was also studied.

\section{Methods}

\section{Subjects}

Forty-five patients, $18-80$ years of age, with moderate to severe COPD admitted to the Centre for Integrated Rehabilitation of Organ Failure (Horn, The Netherlands) for a comprehensive interdisciplinary pulmonary rehabilitation program were included in the study. Patients had to have an acute exacerbation of COPD, which according to the Anthonisen criteria $^{(24)}$ should be treated by a $7 \mathrm{~d}$ antibiotic regimen, as judged by the physician in charge. Exclusion criteria were: immune-compromised subjects or treatment with immune-suppressive medication other than for COPD, pregnancy, lactation, hypersensitivity to the commonly prescribed antibiotics, pre-existing bowel pathology (including irritable bowel syndrome, inflammatory bowel disease, diverticulitis and cancer) and diarrhoea or constipation (in the last $3 \mathrm{~d}$ before inclusion). When using doxycycline maintenance therapy, corticosteroids or gastric acid inhibitors, patients had to be on stable medication for more than 2 weeks. Furthermore, patients were not allowed to use (1) laxatives and anti-diarrhoea medication in the 2 weeks before the start of the study and during the study; (2) regular pro- and prebiotic intake in the 2 weeks before the start of the study and during the study. Finally, ex-smokers had to have quitted smoking for at least 2 weeks before the start of the study.

The present study was conducted according to the guidelines laid down in the Declaration of Helsinki and all procedures involving patients were approved by the Medical Ethics Committee of the University Hospital Maastricht, The Netherlands. Written informed consent was obtained from all the patients.

\section{Study design}

The study was executed according to a parallel, randomised, placebo-controlled, double-blind design. The total duration of the intervention and follow-up period was $63 \mathrm{~d}$. All the patients were prescribed a $7 \mathrm{~d}$ antibiotic treatment and were randomised to receive either $5 \mathrm{~g}$ of a multispecies probiotic, Ecologic ${ }^{\circledR}$ AAD, or placebo twice daily for $14 \mathrm{~d}$ starting simultaneously with antibiotic treatment. This resulted in three time periods defined as: days 1-7, 'the antibiotic/probiotic period' in which all patients received a $7 \mathrm{~d}$ antibiotic treatment in combination with either probiotic or placebo; days 8-14, 'the probiotic only period' in which patients received either probiotic or placebo; days 15-63, 'the post-treatment follow-up period'. The antibiotic treatment was given according to the physician's instructions (care as usual). The time between antibiotic and probiotic intake had to be at least $2 \mathrm{~h}$. Fresh faecal samples were collected on days 1 (the first sample patients were able to produce after inclusion), 7, 14 and 63. On the same day, a questionnaire was filled out including the clinical COPD questionnaire ${ }^{(25)}$, questions on bowel movements (stool frequency and consistency (ranging from ' 1 ', hard lumps, to ' 7 ', completely watery) according to the Bristol stool form scale $\left.{ }^{(26)}\right)$, use of pre- and probiotics, other medication taken, change of eating habits and compliance. In addition, a short questionnaire on bowel habits and sideeffects (nausea, abdominal cramps, bloating, flatulence or other) had to be completed daily during probiotic/placebo intake. Moreover, this questionnaire was used to determine diarrhoea-like defecation defined as a defecation frequency $\geq 3$ /d and/or a faecal consistency (on the Bristol stool form scale) $\geq 5 / \mathrm{d}$ for at least $2 \mathrm{~d}$.

\section{Probiotic}

The multispecies probiotic (Ecologic ${ }^{\circledR}$ AAD) and the placebo were kindly provided by Winclove Bio Industries, Amsterdam, The Netherlands. Ecologic ${ }^{\circledR}$ AAD consists of nine different bacterial species at $10^{8}$ colony forming units/g each, 5\% mineral mix and $15 \%$ Raftilose ${ }^{\circledR}$ Synergy1. All strains are deposited in the Dutch Dairy Institute (NIZO, Ede, The Netherlands) culture collection and are characterised with (GTG)5-PCR fingerprinting as well as further fingerprinting using BOX-A1R and OPY11 primers, followed by SSU (16S)-rRNA sequence analysis (Bifidobacterium bifidum NIZO 3804, Bifidobacterium lactis NIZO 3680 (formerly known as Bifidobacterium longum), Enterococcus faecium NIZO 3886, Lactobacillus acidophilus NIZO 3678 and NIZO 3887, Lactobacillus paracasei NIZO 3672, Lactobacillus plantarum NIZO 3684, Lactobacillus rhamnosus NIZO 3689 and Lactobacillus salivarius NIZO 3675). Individual probiotic 
strains carry the European Union qualified presumption of safety and all the study products (both probiotic and placebo) were prepared under good manufacturing process conditions. Each patient consumed sachets containing $5 \mathrm{~g}$ Ecologic ${ }^{\circledR}$ AAD or placebo twice daily for 2 weeks. Sachets had to be dissolved in lukewarm water, left for $10 \mathrm{~min}$, stirred and thereafter ingested. Both probiotic and placebo were packaged in identical, numbered sachets. The placebo sachets, indistinguishable in colour, smell and taste also contained $5 \%$ mineral mix and $15 \%$ Raftilose ${ }^{\circledR}$ Synergy 1 but contained maizestarch instead of the probiotic bacteria. In a pilot study with five healthy volunteers, the 2-week consumption of $1.5 \mathrm{~g}$ Raftilose ${ }^{\circledR}$ Synergy 1 per day affected neither bifidobacterial communities (using PCR-denaturing gradient gel electrophoresis (DGGE) and most probable number-PCR) nor faecal lactic acid bacteria and lactobacilli counts (CJM Koning, D Jonkers, E Stobberingh, H Smid and $\mathrm{R}$ Stockbrügger, unpublished results).

\section{Sample processing}

Faecal samples were brought to the laboratory within $12 \mathrm{~h}$ after defecation and divided into three portions: (1) $10 \mathrm{~g}$ were centrifuged at $47000 \mathrm{~g}$ for $2 \mathrm{~h}$ at $4{ }^{\circ} \mathrm{C}$ to obtain faecal water, which was frozen immediately in twofold at $-80^{\circ} \mathrm{C}$ for analysis of endotoxin concentrations and determination of $\mathrm{pH}$; (2) $5 \mathrm{~g}$ were diluted (1:4) with peptone water (Oxoid CM9, Basingstoke, Hants, UK) supplemented with cysteine $(2.1 \mathrm{~mm})$ and glycerol $(30 \%)$ and used immediately for bacterial culture; (3) the remaining faecal sample $(1-15 \mathrm{~g})$ was frozen directly at $-20^{\circ} \mathrm{C}$ for molecular analysis.

\section{DNA extraction}

DNA was isolated from approximately $0 \cdot 1 \mathrm{~g}$ of frozen faeces, using the FastDNA SPIN Kit for Soil (Qbiogene, Carlsbad, CA, USA) and a FastPrep Instrument (FP120, Savant Instruments, Farmingdale, NY, USA) following instructions of the manufacturer. Extracted DNA was checked by agarose gel $(1.2 \%(\mathrm{w} / \mathrm{v}))$ electrophoresis in the presence of ethidium bromide.

\section{PCR amplification, denaturing gradient gel electrophoresis analysis and quantitative PCR}

Total bacterial profiles from all the samples were analysed by DGGE of $16 \mathrm{~S}$ rRNA gene fragments amplified by PCR with primers $0968-\mathrm{f}-\mathrm{GC}$ and $1401-\mathrm{r}$ as described previously ${ }^{(27,28)}$. Amplicons generated by PCR were separated by DGGE in $8 \%$ polyacrylamide gels using a DCode TM system (Bio-Rad Laboratories, Hercules, CA, USA) as described elsewhere ${ }^{(28,29)}$. For the separation of amplicons, gradients of $40-50 \%$ were used ( $100 \%$ denaturing solution was defined as $7 \mathrm{M}$-urea and $40 \%$ formamide). Gels were stained with $\mathrm{AgNO}_{3}^{(30)}$. The DGGE gels were analysed using Bionumerics 4.0 (Applied Maths BVBA, Sint-Martens-Latem, Belgium). Following normalisation of gel images using markers loaded on either side and in the central lane of each gel, bands were defined for each sample using the bands searching algorithm within the program. Corresponding densitometric curves were used for a manual check of band definition, and bands with $<1 \%$ of the total area of all bands were omitted from further analysis ${ }^{(31)}$. The similarity of the DGGE profiles (expressed as similarity indices (SI)) with baseline (day 0; e.g. $0-7,0-14$, etc.) and between consecutive days (e.g. 0-7, 7-14, etc.) was determined by calculating a Dice correlation coefficient (band based) according to the principle of moving window correlation ${ }^{(32)}$.

Quantitative detection of total bacteria, lactobacilli and bifidobacteria was performed using an iCycler IQ real-time detection system associated with the iCycler optical system interface software version 2.3 (Bio-Rad, Veenendaal, The Netherlands). Total bacterial 16S rRNA gene copies were quantified with primers Bact-1369-f and Prok-1492-r ${ }^{(33)}$, lactobacilli were quantified using primers LactoF and LactoR $^{(34)}$ and bifidobacteria by primers Bif 164-f and $662-r^{(35)}$ as described previously ${ }^{(36)}$.

\section{Bacteriological culture}

Microbiological analysis of the faecal samples was determined as previously described ${ }^{(37)}$. Briefly, tenfold serial dilutions of the faecal dilution were made in physiological saline $(0.85 \%)$ with cysteine- $\mathrm{HCl}(0.05 \%)$, and $40 \mu \mathrm{l}$ of these dilutions were inoculated on agar plates to culture and count total (facultative) aerobic bacteria (blood agar, Oxoid CM 271), enterobacteria (methylthionium chloride agar, Oxoid CM69), enterococci (KF-streptococcus agar, Oxoid CM701), total (facultative) anaerobic bacteria (fastidious anaerobic agar, Laboratory $\mathrm{M}$ LabGo), Bacteroides spp. (Bile Esculin agar, Becton Dickinson 287920), lactobacilli (LAMVAB agar ${ }^{(38)}$ ) and yeasts (Sabouraud GM $+\mathrm{C}$ agar, Becton Dickinson 254041). Escherichia coli and enterococci isolates were identified using standard biochemical tests ${ }^{(39)}$ and stored at $-80^{\circ} \mathrm{C}$ for susceptibility testing.

Table 1. Patient characteristics*

(Mean values and standard deviations)

\begin{tabular}{|c|c|c|c|c|}
\hline & \multicolumn{2}{|c|}{ Probiotic ( $n$ 17) } & \multicolumn{2}{|c|}{ Placebo $(n 13)$} \\
\hline & Mean & SD & Mean & SD \\
\hline $\operatorname{Sex} \mathrm{m} / \mathrm{f}(n)$ & $12 / 5$ & & $7 / 6$ & \\
\hline Age (years) & $59 \cdot 9$ & $13 \cdot 3$ & 63.4 & 7.4 \\
\hline Current smokers $(n)$ & 3 & & 1 & \\
\hline BMI $\left(\mathrm{kg} / \mathrm{m}^{2}\right)$ & $27 \cdot 1$ & $6 \cdot 6$ & $27 \cdot 4$ & 4.9 \\
\hline FFMI $\left(\mathrm{kg} / \mathrm{m}^{2}\right)$ & $17 \cdot 7$ & $2 \cdot 6$ & 18.4 & $5 \cdot 7$ \\
\hline $\mathrm{FEV}_{1}(\%$ pred $)$ & 43.0 & $20 \cdot 3$ & $40 \cdot 8$ & $28 \cdot 0$ \\
\hline FVC (\% pred) & $84 \cdot 7$ & $17 \cdot 1$ & $75 \cdot 6$ & $19 \cdot 5$ \\
\hline CCQ total score & $3 \cdot 0$ & 0.7 & 3.25 & 0.9 \\
\hline $\begin{array}{l}\text { Antibiotic treatments } \\
\text { in previous year }\end{array}$ & 2.9 & $2 \cdot 6$ & $3 \cdot 3$ & $2 \cdot 1$ \\
\hline $\begin{array}{l}\text { Use of pre- and probiotics } \\
\text { before the study }(n)\end{array}$ & 0 & & $1 \dagger$ & \\
\hline $\begin{array}{l}\text { Compliance } \neq \text { probiotic/placebo } \\
\text { intake (\%) }\end{array}$ & $98 \cdot 1$ & 4.6 & $99 \cdot 7$ & 1.0 \\
\hline
\end{tabular}

$\mathrm{m} / \mathrm{f}$, Male/female; FFMI, fat free mass index; $F E V_{1}$, forced expiratory volume in $1 \mathrm{~s}$; FVC, forced vital capacity; CCQ, clinical chronic obstructive pulmonary disease questionnaire; dd, developmentally disabled.

* There was no significant difference between the probiotic and the placebo groups $(P>0.05)$.

$\dagger$ Yakult 1 dd.

$¥$ Compliance is self-reported. 


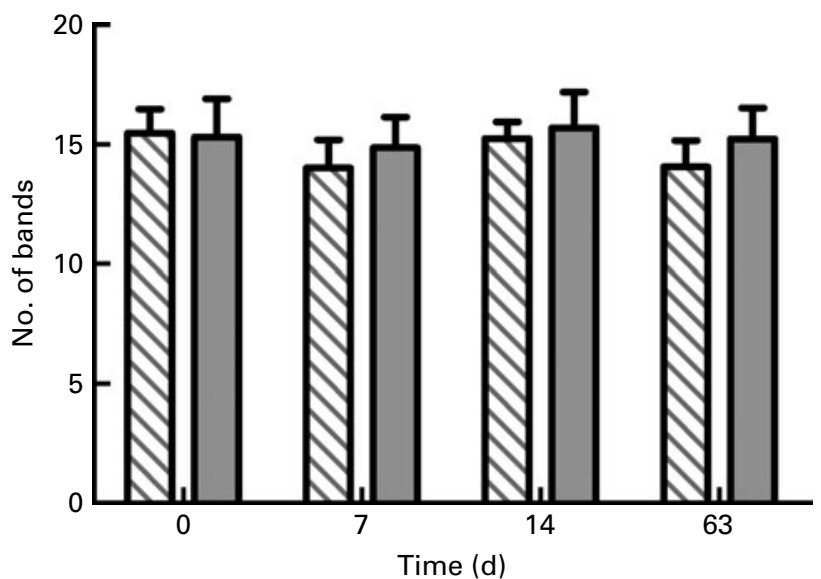

Fig. 1. Total bacterial band richness (mean (SEM) no. of bands) obtained from denaturing gradient gel electrophoresis profiles of probiotic- $(\mathbb{\mathbb { Q }}$ ) and placebo $(\square)$-treated patients before, during (days 1-7) and after antibiotic intake.

\section{Susceptibility testing}

Antimicrobial susceptibilities (as minimal inhibitory concentration values) for the $E$. coli and enterococci isolates were determined using the microbroth dilution method with cation-adjusted Mueller-Hinton broth II (Becton, Dickinson and Company, Sparks, MD, USA) according to the Clinical and Laboratory Standards Institute (formerly the National Committee for Clinical Laboratory Standards) guidelines as described previously ${ }^{(40)}$. Microtitre plates containing freezedried antibiotics were obtained from MCS Diagnostics B.V. (Swalmen, The Netherlands) after quality testing by the manufacturer and a guaranteed shelf-life of about 1 year. The minimal inhibitory concentration was defined as the lowest concentration showing no growth in the microtitre plates after $18 \mathrm{~h}$ of incubation at $37^{\circ} \mathrm{C}$. E. coli ATCC 35218 and ATCC 25922 and Escherichia faecalis ATCC 29212 were used as quality control reference strains.

\section{Endotoxin and $\mathrm{pH}$}

The endotoxin (i.e. lipopolysaccharide) concentration was determined in faecal water using the Limulus amoebocyte lysate endochrome technique (Endosafe, end-point chromogenic analysis endochrome test kit; Charles River, Kent, UK), according to the manufacturer's specifications under pyrogen-free conditions. Pyrogen-free water was used to dilute the faecal samples, the test solutions, and was used as a negative control. The detection range of the assay was 0.015-0.12 endotoxin units/ml (9 endotoxin units/ng). Concentration of faecal endotoxin was expressed as nanogram of endotoxin per $\mathrm{ml}$ of faecal water. The $\mathrm{pH}$ of faecal water was determined using a PHM standard $\mathrm{pH}$ meter with a PHC3006 electrode (Radiometer Nederland B.V., Zoetermeer, The Netherlands).

\section{Statistics}

The treatment allocation was concealed to all investigators and volunteers, until the study had been completed and all the analyses had been performed.

The primary outcome of the present study was to assess the restoration of the dominant intestinal microbiota after antibiotic treatment in probiotic- and placebo-treated patients by PCR-DGGE. Based on data from our previous study in healthy volunteers ${ }^{(10)}$, it was estimated that fourteen volunteers per treatment group would provide a $80 \%$ power to detect a $20 \%$ increase in SI after disturbance of antibiotic intake, assuming a variance of $18 \%$ and a two-sided significance level of $0 \cdot 05$.

Secondary outcomes were to assess changes in probioticand placebo-treated patients during and after antibiotic intake in (1) specific bacterial subgroups, using both culturedependent and molecular based techniques; (2) bowel habits; (3) the incidence of antibiotic resistance; (4) endotoxin concentration and $\mathrm{pH}$. In addition, the disturbance of the dominant intestinal microbiota in the subjects who develop AAD compared with those who did not was assessed.

Statistical evaluation of DGGE band richness, quantitative PCR, culture, endotoxin concentration and $\mathrm{pH}$ was carried out using linear mixed model analysis as described previously $^{(10,37)}$.

For all other data the nonparametric Mann-Whitney $U$ test was used for two-group comparisons of independent ordinal and interval values, while the nonparametric Wilcoxon signed-ranked test was used for comparison of related ordinal and interval values. If the data were normally distributed, the Student's $t$-test was used. For dichotomous variables, Pearson's $\chi^{2}$-test was applied to test for differences between groups. All the tests were conducted using SPSS version 11.0 (SPSS, Inc., Chicago, IL, USA) and a $P$ value below 0.05 was considered statistically significant.

Table 2. Similarity indices $(\mathrm{SI})$ of the total bacterial profiles in percentages in the probiotic group $v$. the placebo group compared with baseline $(t=0)$ and between consecutive intervals

(Median and range values)

\begin{tabular}{|c|c|c|c|c|c|c|c|c|c|c|}
\hline & \multicolumn{10}{|c|}{ SI (\%) } \\
\hline & \multicolumn{2}{|c|}{$0-7$} & \multicolumn{2}{|c|}{$0-14$} & \multicolumn{2}{|c|}{$0-63$} & \multicolumn{2}{|c|}{$7-14$} & \multicolumn{2}{|c|}{$14-63$} \\
\hline & Median & Range & Median & Range & Median & Range & Median & Range & Median & Range \\
\hline Probiotic $^{\star}(n$ 17) & 90 & $69-98$ & 87 & $46-96$ & 88 & $65-95$ & 90 & $56-98$ & 90 & $63-98$ \\
\hline Placebo $(n 13)$ & 94 & $44-98$ & 93 & $45-98$ & 84 & $39-97$ & 91 & $69-98$ & 89 & $55-98$ \\
\hline
\end{tabular}

${ }^{*}$ Within group decrease $0-63$ v. 0-7, $P<0.05$. 


\section{Results}

\section{Subjects}

From September 2005 to December 2006, forty-five patients were enrolled of which thirty patients completed the study; seventeen in the probiotic group and thirteen in the placebo group. Eleven patients $(73 \%)$ discontinued the study in the first week, three patients $(20 \%)$ in the second week and one patient $(7 \%)$ thereafter. The most common reasons for discontinuation included 'not being able or willing to collect faeces'; 'perception of the study load as being too demanding considering the current medical condition'; 'not liking the taste of the study product'. Baseline demographics and clinical characteristics were comparable for both the groups (Table 1). The antibiotic treatments prescribed for the exacerbation were mostly doxycycline and amoxycillin/clavulanic acid and were also comparable for both the groups. The mean frequency of antibiotic use was $3 \cdot 1$ (SD 2.4) treatments in the year before inclusion.

Eight patients in the probiotic group and five patients in the placebo group $(P=0.72)$ changed their dietary habits during the study period, mostly based on medical indication (energy restriction ( $n$ 10), supplementation with nutrient drinks ( $n$ 2), loss of appetite $(n 1))$. Apart from the exclusion criteria, patients in both the groups used diverse and extensive medication mostly belonging to the groups of analgesia, antidepressants, antihypertensives, diuretics, sympathicomimetics, xanthines and parasympathicolytics.

There were no reported adverse events related to the study product. Compliance was assessed by the self-reported number of study product (sachets) that was consumed.

\section{Bowel habits}

The mean defecation frequency (probiotic, 1.4 (SEM 0.4), $2 \cdot 0$ (SEM 0.4), 1.6 (SEM 0.3); placebo, 1.1 (SEM 0.1), 1.4 (SEM 0.2 ), 1.6 (SEM 0.3)) and consistency (probiotic, 3.9 (SEM 0.4 ), 4.5 (SEM 0.3), 4.6 (SEM 0.2); placebo 3.5 (SEM 0.4), 4.2 (SEM 0.4), $4.0($ SEM 0.4)) before antibiotic and probiotic intake (day 0 , i.e. baseline), during the antibiotic/probiotic period (days 1-7) and during the probiotic only period (days 8-14), respectively, did not differ significantly between the probiotic and the placebo groups. In the probiotic group, the defecation frequency during antibiotic intake was significantly higher (2.0 (SEM 0.4)) compared with baseline (1.4 (SEM 0.4); $P<0.05$ ). During and 1 week after antibiotic intake (days 1-14), diarrhoea-like bowel movements were reported frequently in both the probiotic group (77\%) and the placebo group $(70 \% ; P>0.05)$.

\section{PCR-denaturing gradient gel electrophoresis analysis of the dominant faecal microbiota}

DGGE analysis of the dominant faecal microbiota showed high inter-individual variation in total bacterial profiles between patients and a mean number of 15.4 bands per profile (i.e. band richness) at the start of the study (day 0). No difference in band richness between the probiotic and the placebo groups or over time within both the groups was found (Fig. 1).
SI were high and remained stable during and after antibiotic treatment (Table 2). No effect of probiotic intake was observed. At day 63, lower SI (compared with baseline) were found in both the groups, which were only significant in the probiotic group. Moreover, no association between the SI of the total bacterial profiles during amoxycillin and probiotic intakes and the occurrence of diarrhoea-like bowel movements was observed (data not shown).
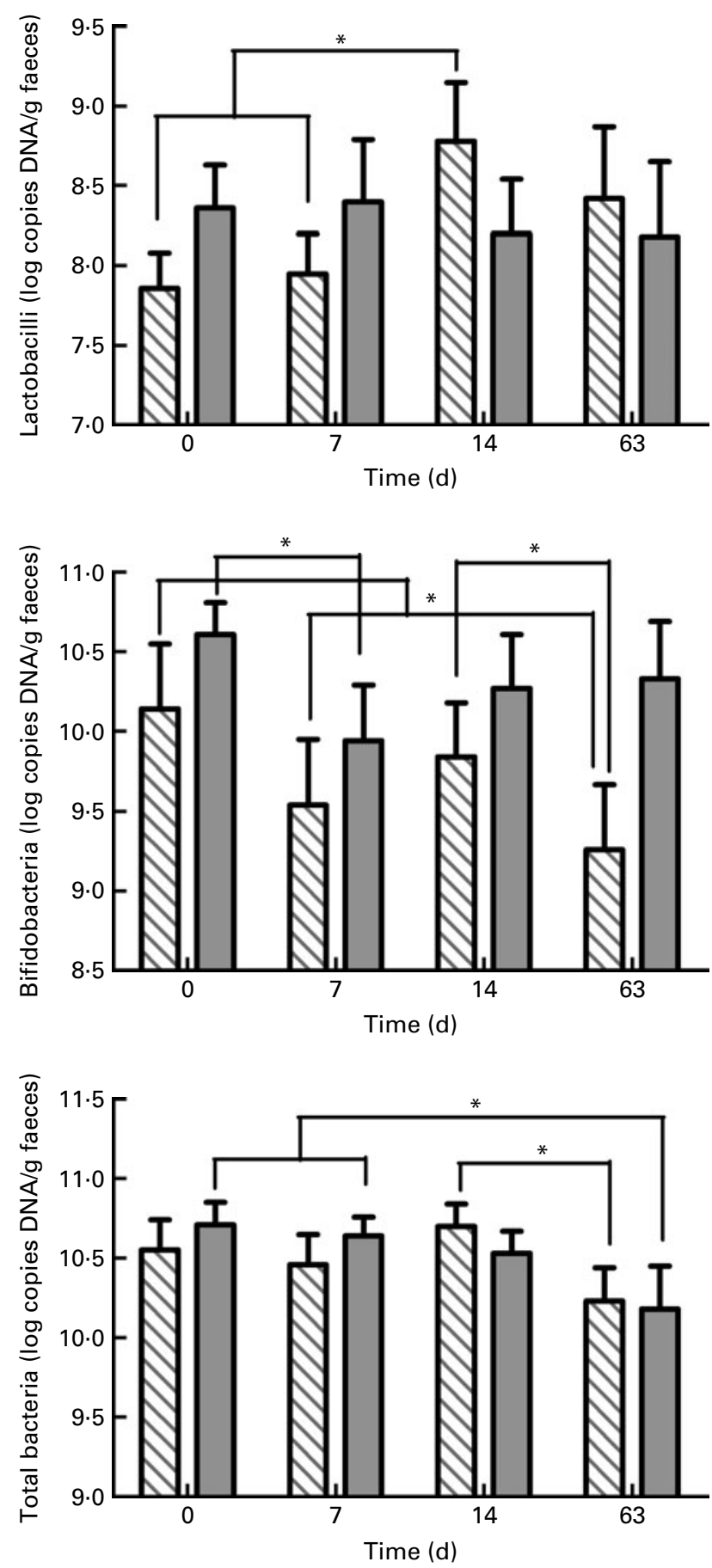

Fig. 2. Quantitative PCR results expressed as mean (SEM) log copies DNA/g faeces obtained from faeces of probiotic- $(\mathbb{Q})$ and placebo $(\square)$-treated patients before, during (days $1-7$ ) and after antibiotic intake: ${ }^{\star} P<0.05$. 


\section{Quantitative PCR}

No differences in the 16S rRNA gene copy numbers of total bacteria, lactobacilli or bifidobacteria were observed between the probiotic and the placebo groups. Within both the groups, a significant decrease of bifidobacteria was found during antibiotic intake, which increased again after cessation (Fig. 2). During probiotic intake (day 14), a small increase in total bacteria was observed in the probiotic group, which significantly decreased again after cessation. Moreover, a significant increase in lactobacilli was observed in the probiotic group (Fig. 2). For all the bacterial species studied, values on day 63 did not differ significantly from day 0 apart from a decrease in bifidobacteria in the probiotic group and a decrease in total bacteria in the placebo group (Fig. 2). To adjust for possible differences in faecal consistency, data were also analysed as $16 \mathrm{~S}$ rRNA gene copies per $\mathrm{mg}$ faecal protein with similar results compared with the unadjusted data (data not shown).

\section{Bacteriological culture}

No differences in either aerobic or anaerobic culturedbacterial species were observed between the probiotic and the placebo groups (Table 3). However, group-specific differences were observed over time. During 'the probiotic only period (14d)', the mean number of faecal enterococci increased significantly within the probiotic group, returning to pre-treatment level 7 weeks after cessation $(P<0.05$; Table 3). Moreover, within the probiotic group, a significant

(Mean values with their standard errors) decrease was found in enterobacteria (day $7 v$. day 0 ), and significant increases were observed over time in total aerobes (days 7 and $14 v$. days 0 and 63), Bacteroides spp. (day $14 v$. days 0 and 63) and lactobacilli (days 14 and $63 v$. day 7) $(P<0 \cdot 05)$. Within both the groups, a significant increase in yeast was found during antibiotic intake (day 7; Table 3 ).

For all the bacterial species, in both the groups, bacterial counts on day 63 did not differ significantly from day 0.

\section{Antimicrobial susceptibility}

E. coli isolates were isolated in $47-82 \%$ (probiotic) and 54-69\% (placebo) of the faecal samples and enterococci were isolated in 77-100\% (probiotic) and 92\% (placebo) of the faecal samples from the patients at the different points of time. No significant differences in antibiotic susceptibility of $E$. coli and enterococci were observed.

\section{Endotoxin and $\mathrm{pH}$}

Mean endotoxin concentrations, expressed as log $\mathrm{ng} / \mathrm{ml}$ faecal water, did not differ significantly between groups and within both groups during the study period (probiotic, 2.5 (SEM 0.1), 2.6 (SEM 0.08), 2.5 (SEM 0.07) and 2.4 (SEM 0.09); placebo, 2.5 (SEM 0.06), 2.5 (SEM 0.09), 2.5 (SEM 0.09), 2.5 (SEM 0.01) at $t=0,7,14$ and 63, respectively). Also, no significant correlation between the number of enterobacteria cultured and endotoxin concentration was found.

Table 3. Numbers of bacteria cultured expressed as log colony-forming units/g faeces

\begin{tabular}{|c|c|c|c|c|c|c|c|c|}
\hline & \multicolumn{2}{|c|}{ Day 0} & \multicolumn{2}{|c|}{ Day 7} & \multicolumn{2}{|c|}{ Day 14} & \multicolumn{2}{|c|}{ Day 63} \\
\hline & Mean & SEM & Mean & SEM & Mean & SEM & Mean & SEM \\
\hline \multicolumn{9}{|c|}{ Total aerobic microbiota } \\
\hline Probiotic* & 7.4 & 0.2 & $8 \cdot 2$ & 0.3 & $8 \cdot 4$ & 0.2 & $7 \cdot 4$ & 0.3 \\
\hline Placebo & 7.5 & 0.4 & $7 \cdot 3$ & 0.3 & 7.5 & 0.3 & 7.4 & 0.3 \\
\hline \multicolumn{9}{|c|}{ Enterobacteriaceae } \\
\hline Probiotic $†$ & 6.5 & 0.4 & $4 \cdot 6$ & 0.8 & $5 \cdot 3$ & 0.7 & $5 \cdot 3$ & $0 \cdot 6$ \\
\hline Placebo & 5.5 & 0.7 & $4 \cdot 1$ & 0.8 & 4.5 & 0.8 & 5.9 & 0.6 \\
\hline \multicolumn{9}{|l|}{ Enterococci } \\
\hline Probioticł & $5 \cdot 5$ & 0.4 & $5 \cdot 8$ & 0.5 & $7 \cdot 1$ & 0.4 & 4.9 & 0.5 \\
\hline Placebo & $5 \cdot 3$ & 0.5 & $6 \cdot 4$ & 0.4 & $5 \cdot 7$ & 0.6 & 5.4 & 0.4 \\
\hline \multicolumn{9}{|c|}{ Total anaerobic microbiota } \\
\hline Probiotic & 9.5 & 0.2 & $9 \cdot 1$ & 0.5 & $9 \cdot 1$ & 0.5 & $9 \cdot 2$ & $0 \cdot 2$ \\
\hline Placebo & 9.5 & $0 \cdot 1$ & 9.5 & 0.2 & $9 \cdot 1$ & 0.7 & $9 \cdot 8$ & $0 \cdot 1$ \\
\hline \multicolumn{9}{|c|}{ Bacteroides spp. } \\
\hline Probiotic§ & $7 \cdot 2$ & 0.3 & $7 \cdot 6$ & 0.4 & $8 \cdot 1$ & 0.2 & $7 \cdot 2$ & $0 \cdot 3$ \\
\hline Placebo & $7 \cdot 0$ & 0.5 & 7.9 & 0.2 & $7 \cdot 8$ & 0.3 & $7 \cdot 8$ & $0 \cdot 2$ \\
\hline \multicolumn{9}{|l|}{ Lactobacilli } \\
\hline Probiotic\| & $5 \cdot 3$ & 0.4 & $5 \cdot 1$ & 0.5 & $6 \cdot 0$ & 0.5 & 5.9 & 0.5 \\
\hline Placebo & $5 \cdot 3$ & 0.6 & $5 \cdot 1$ & 0.4 & $5 \cdot 7$ & 0.5 & $5 \cdot 6$ & 0.5 \\
\hline \multicolumn{9}{|l|}{ Yeast } \\
\hline Probiotic & $2 \cdot 8$ & 0.4 & 3.9 & 0.4 & $3 \cdot 2$ & 0.3 & $3 \cdot 2$ & 0.4 \\
\hline Placebo** & $2 \cdot 2$ & 0.4 & $3 \cdot 2$ & 0.4 & $2 \cdot 9$ & 0.4 & $2 \cdot 2$ & 0.3 \\
\hline
\end{tabular}

* Within group increase $t=7 / 14 v . t=0 / 63, P<0.05$.

tWithin group decrease $t=7 \mathrm{v} . t=0, P<0.05$.

$\ddagger$ Within group increase $t=14 \mathrm{v}, t=0 / 7 / 63, P<0.05$

$\S$ Within group increase $t=14$ v. $t=0 / 63, P<0.05$.

$\|$ Within group increase $t=14 / 63$ v. $t=7, P<0.05$

I Within group increase $t=7 \mathrm{v} . t=0, P<0.05$

${ }^{* *}$ Within group increase $t=7 \mathrm{v} . t=0 / 63, P<0.05$. 
The $\mathrm{pH}$ of faecal water ranged from 6.3 to 6.7 and did not differ between groups and within both groups during the study period.

\section{Discussion}

The present double-blind, placebo-controlled, randomised study demonstrated that the intake of a multispecies probiotic in this COPD population with a history of frequent antibiotic use had no effect on the composition of the dominant faecal microbiota. Moreover, in contrast to previous findings in healthy volunteers, no effect of antibiotic intake on the composition of the dominant faecal microbiota could be found and no difference in SI was observed between the group of patients who developed diarrhoea-like bowel movements and those who did not. Looking at specific bacterial subgroups, using both molecular and culture-dependent techniques, a significant but small antibiotic effect was observed in both the groups, i.e. an increase in yeasts and a decrease in bifidobacteria. No differences were observed when comparing the probiotic group with the placebo group. However, changes in specific bacterial groups over time were seen in the probiotic group only, suggesting a modest probiotic effect.

In healthy human subjects, the faecal microbiota is host specific and relatively stable over time $(\mathrm{SI}>80 \%)^{(5,6)}$. It is well documented that despite methodological differences, antibiotic treatment, especially amoxycillin, cephalosporins, clindamycin or broad-spectrum antibiotics, has a marked effect on the composition of the intestinal microbiota ${ }^{(8,41,42)}$, which may result in diarrhoea. In line with these findings, we have previously shown that amoxycillin intake strongly affected the composition of the dominant faecal microbiota in healthy volunteers, indicated by a low mean SI $(<52 \%)$ and a decrease in bacterial richness (i.e. number of bands). We also showed that a lower stability of the dominant intestinal microbiota (i.e. more disruption) is related to a higher risk of developing $\mathrm{AAD}^{(10)}$. Remarkably, using the same methods, in the COPD population mean SI were high $(>90 \%)$ and both the SI and the band richness remained stable during and after antibiotic intake. Moreover, no difference in SI (i.e. disruption) was observed in the group of patients, who developed diarrhoea-like bowel movements compared with those who did not. This unexpected lack of effect on the microbiota could be attributed to the frequent antibiotic use in the past by this population. Recently, it has been shown that antibiotic intake can cause medium and long-term disturbances in (specific) bacterial populations ${ }^{(9,10)}$. Moreover, as antibiotics only affect sensitive strains, extensive antibiotic use could have caused a prolonged narrowing of the diversity into a dominant faecal microbiota consisting of microbial populations insensitive to the given antibiotics. This assumption is further supported by the fact that bacterial band richness in COPD patients at the start of the study was relatively low, i.e. being at the same level as in healthy volunteers during amoxycillin intake, in whom band richness was significantly reduced ${ }^{(10)}$. It is known that the bacterial diversity decreases with age ${ }^{(43)}$, and we readily acknowledge that the mean age of the patients in the present study was significantly higher than that of the healthy volunteers in the previous study. However, this cannot account for the fact that no antibiotic effect on bacterial diversity was observed in the COPD patients. In fact, this finding was corroborated by a similar trend in endotoxin concentrations. Previously, antibiotics have been shown to increase the bioavailability of endotoxin originating from gram-negative bacteria ${ }^{(4-47)}$. However, in our study, we found that endotoxin concentrations remained stable during and after antibiotic intake and were even slightly higher than the levels found in healthy volunteers during amoxycillin intake, where an increase was found ${ }^{(37)}$.

In addition to qualitative data on microbiota composition obtained by DGGE analysis of 16S rRNA gene fragments, quantitative analysis of specific bacterial subgroups was performed by culture and quantitative PCR.

With culture, apart from a small but clinically insignificant rise in yeasts, no changes were observed, that could be attributed to antibiotic intake. Moreover, with quantitative PCR, no effect of antibiotic intake was found on total bacteria, which is in line with the DGGE results. This further supports the lack of observed antibiotic effect. However, the number of bifidobacteria was significantly affected in both the groups during antibiotic intake corresponding with the reported high susceptibility of bifidobacteria to broad-spectrum antibiotics ${ }^{(48-51)}$.

Various effects over time were observed in the probiotic group only, which can be attributed to probiotic intake. The increase in faecal enterococci and lactobacilli during probiotic intake is in line with literature showing that the intake of a probiotic is able to transiently increase the faecal number of the ingested strains ${ }^{(52-54)}$. We previously showed that the consumption of this multispecies probiotic, containing E. faecium, was associated with a significant increase in the concentration of faecal enterococci in the probiotic group and that the pulsed-field gel electrophoresis patterns of the enterococci cultured were similar to the orally administered E. faecium $^{(37)}$. Apart from lactobacilli and the E. faecium, the multispecies probiotic also contained bifidobacteria. However, no increase in bifidobacteria could be observed, probably due to the fact that the administered daily amount $\left(2 \times 10^{9}\right.$ colony forming units) was too low in relation to the baseline value.

The multispecies product was well tolerated and no adverse events related to the product were reported. The majority of literature shows that the probiotics can significantly reduce the relative risk of developing $\mathrm{AAD}^{(19,20,22)}$. In contrast, in the present study, the intake of the multispecies probiotic did not result in a decrease in diarrhoea-like bowel movements. However, earlier we showed, in healthy volunteers, that the intake of the same multispecies probiotic had a restoring and stabilising impact on the dominant faecal microbiota after amoxycillin intake ${ }^{(10)}$. A comparable observation was made by Kajander et al. ${ }^{(5)}$, when a multispecies probiotic was given to irritable bowel syndrome patients. In the present study, antibiotic intake did not affect the composition of the dominant microbiota, probably contributing to the lack of effect of probiotic intake on the occurrence of diarrhoea-like bowel movements in this COPD population. Furthermore, as the microbiota seems to be profoundly altered by the frequent prior antibiotic use, a much longer probiotic treatment might be required to restore such an effect.

We acknowledge that the present study has some limitations. Inherent to the nature and severity of the disease, diverse and extensive medication was used in this COPD population, and we cannot exclude that some medication had an impact on the composition of the microbiota. 
Nevertheless, patients had to be on stable medication for corticosteroids, gastric acid inhibitors and doxycycline maintenance therapy before and during the study to limit this effect. Although we clearly showed no effect of antibiotic treatment on the intestinal microbiota due to the heterogeneity of the population and the limited sample size, subtle changes in subgroups' analyses may have failed to be detected.

Currently, it is widely accepted that antibiotic treatment profoundly affects the intestinal microbiota. However, our findings show that the dominant faecal microbiota was not affected upon antibiotic treatment in COPD patients with a history of frequent antibiotic use. This prolonged antibiotic pressure seems to have caused a long-term imbalance of the microbiota with a shift toward populations resistant to antibiotics. Furthermore, the present study demonstrates that the short-term intake of a multispecies probiotic had a modest effect on the intestinal microbiota, albeit much smaller than previously observed for healthy adults. Nevertheless, this did not result in a restoration of the microbiota imbalance and as a result no reduction in diarrhoea-like bowel movements was found.

These results warrant further confirmation in studies with a higher number of patients with higher doses of probiotics and a longer administration period.

In future studies, it would be very interesting to determine to what extent these disturbances are permanent, what the effect of these disturbances is and if probiotic supplementation is able to either prevent or with prolonged use reverse such an imbalance.

\section{Acknowledgements}

The authors would like to thank Annie van de Kruijs (Centre for Integrated Rehabilitation of Organ failure, Horn, The Netherlands) for expert assistance with the enrolment of patients and collection of samples, and Wilma Akkermansvan Vliet and Ineke Heikamp-de Jong (Laboratory of Microbiology, Wageningen University, The Netherlands) for excellent assistance with the PCR-DGGE analyses. The present study was supported by SenterNovem, an agency of the Dutch Ministry of Economic Affairs (grant no. TSGE 1041). R. S. is the guarantor of the article. C. K. was the principal investigator and conducted the study. C. K., D. J. and H. S. were responsible for the analyses of the study. All the authors participated in the design, execution and data interpretation of the study and contributed significantly to the various drafts of the manuscript. All the authors approved the final draft submitted. F. R. is an advisor of Winclove Bio Industries B.V. C. K. is supported, in part, by the grant from SenterNovem and, in part, by Winclove Bio Industries B.V. All the other authors declare they have no conflict of interest.

\section{References}

1. Rajilic-Stojanovic M, Smidt H \& de Vos WM (2007) Diversity of the human gastrointestinal tract microbiota revisited. Environ Microbiol 9, 2125-2136.

2. Turnbaugh PJ, Hamady M, Yatsunenko T, et al. (2008) A core gut microbiome in obese and lean twins. Nature 457, 480-484.

3. Zoetendal EG, Vaughan EE \& de Vos WM (2006) A microbial world within us. Mol Microbiol 59, 1639-1650.
4. Maukonen J, Matto J, Satokari R, et al. (2006) PCR DGGE and RT-PCR DGGE show diversity and short-term temporal stability in the Clostridium coccoides-Eubacterium rectale group in the human intestinal microbiota. FEMS Microbiol Ecol 58, 517-528.

5. Vanhoutte T, Huys G, De brandt E, et al. (2004) Temporal stability analysis of the microbiota in human feces by denaturing gradient gel electrophoresis using universal and group-specific 16S rRNA gene primers. FEMS Microbiol Ecol 48, 437-446.

6. Zoetendal EG, Akkermans AD \& De Vos WM (1998) Temperature gradient gel electrophoresis analysis of $16 \mathrm{~S}$ rRNA from human fecal samples reveals stable and hostspecific communities of active bacteria. Appl Environ Microbiol 64, 3854-3859.

7. Zoetendal EG, Rajilic-Stojanovic M \& de Vos WM (2008) High-throughput diversity and functionality analysis of the gastrointestinal tract microbiota. Gut 57, 1605-1615.

8. Dethlefsen L, Huse S, Sogin ML, et al. (2008) The pervasive effects of an antibiotic on the human gut microbiota, as revealed by deep $16 \mathrm{~S}$ rRNA sequencing. PLoS Biol 6, e280.

9. Jernberg C, Lofmark S, Edlund C, et al. (2007) Long-term ecological impacts of antibiotic administration on the human intestinal microbiota. ISME J 1, 56-66.

10. Koning CJM, Jonkers DMAE, Stobberingh EE, et al. (2005) Effect of a multispecies probiotic on the composition of the dominant faecal flora in healthy volunteers. Gut 54, Suppl. VII, A234.

11. Barnes PJ (2000) Mechanisms in COPD: differences from asthma. Chest 117, 10S-14S.

12. Turato G, Zuin R \& Saetta M (2001) Pathogenesis and pathology of COPD. Respiration 68, 117-128.

13. Burge S \& Wedzicha JA (2003) COPD exacerbations: definitions and classifications. Eur Respir J Suppl 41, 46s-53s.

14. Donaldson GC, Seemungal TA, Patel IS, et al. (2003) Longitudinal changes in the nature, severity and frequency of COPD exacerbations. Eur Respir J 22, 931-936.

15. Cebra JJ (1999) Influences of microbiota on intestinal immune system development. Am J Clin Nutr 69, 1046S-1051S.

16. Hogenauer C, Hammer HF, Krejs GJ, et al. (1998) Mechanisms and management of antibiotic-associated diarrhea. Clin Infect Dis 27, 702-710.

17. Macpherson AJ \& Uhr T (2004) Induction of protective IgA by intestinal dendritic cells carrying commensal bacteria. Science 303, 1662-1665.

18. Noverr MC \& Huffnagle GB (2004) Does the microbiota regulate immune responses outside the gut? Trends Microbiol 12, 562-568

19. Cremonini F, Di Caro S, Nista EC, et al. (2002) Meta-analysis: the effect of probiotic administration on antibiotic-associated diarrhoea. Aliment Pharmacol Ther 16, 1461-1467.

20. D'Souza AL, Rajkumar C, Cooke J, et al. (2002) Probiotics in prevention of antibiotic associated diarrhoea: meta-analysis. BMJ 324, 1361

21. Hickson M, D'Souza AL, Muthu N, et al. (2007) Use of probiotic Lactobacillus preparation to prevent diarrhoea associated with antibiotics: randomised double blind placebo controlled trial. BMJ 335, 80.

22. McFarland LV (2006) Meta-analysis of probiotics for the prevention of antibiotic associated diarrhea and the treatment of Clostridium difficile disease. Am J Gastroenterol 101, 812-822.

23. Timmerman HM, Niers LE, Ridwan BU, et al. (2007) Design of a multispecies probiotic mixture to prevent infectious complications in critically ill patients. Clin Nutr 26, 450-459.

24. Anthonisen NR, Manfreda J, Warren CP, et al. (1987) Antibiotic therapy in exacerbations of chronic obstructive pulmonary disease. Ann Intern Med 106, 196-204. 
25. van der Molen T, Willemse BW, Schokker S, et al. (2003) Development, validity and responsiveness of the clinical COPD questionnaire. Health Qual Life Outcomes 1, 13.

26. O'Donnell LJ, Virjee J \& Heaton KW (1990) Detection of pseudodiarrhoea by simple clinical assessment of intestinal transit rate. BMJ 300, 439-440.

27. Nubel U, Engelen B, Felske A, et al. (1996) Sequence heterogeneities of genes encoding 16S rRNAs in Paenibacillus polymyxa detected by temperature gradient gel electrophoresis. $J$ Bacteriol 178, 5636-5643.

28. Sousa DZ, Pereira MA, Stams AJ, et al. (2007) Microbial communities involved in anaerobic degradation of unsaturated or saturated long-chain fatty acids. Appl Environ Microbiol 73, 1054-1064.

29. Muyzer G, de Waal EC \& Uitterlinden AG (1993) Profiling of complex microbial populations by denaturing gradient gel electrophoresis analysis of polymerase chain reaction-amplified genes coding for 16S rRNA. Appl Environ Microbiol 59, $695-700$.

30. Sanguinetti CJ, Dias Neto E \& Simpson AJ (1994) Rapid silver staining and recovery of PCR products separated on polyacrylamide gels. Biotechniques 17, 914-921.

31. Konstantinov SR, Zhu W-Y, Williams BA, et al. (2003) Effect of fermentable carbohydrates on faecal bacterial communities as revealed by DGGE analysis of $16 \mathrm{~S}$ rDNA. FEMS Microbiol Ecol 43, 225-235.

32. Possemiers S, Verthe K, Uyttendaele S, et al. (2004) PCR-DGGE-based quantification of stability of the microbial community in a stimulator of the human intestinal microbial ecosystem. FEMS Microbiol Ecol 49, 495-507.

33. Suzuki MT, Taylor LT \& DeLong EF (2000) Quantitative analysis of small-subunit rRNA genes in mixed microbial populations via $5^{\prime}$-nuclease assays. Appl Environ Microbiol 66, 4605-4614.

34. Byun R, Nadkarni MA, Chhour KL, et al. (2004) Quantitative analysis of diverse Lactobacillus species present in advanced dental caries. J Clin Microbiol 42, 3128-3136.

35. Satokari RM, Vaughan EE, Akkermans AD, et al. (2001) Bifidobacterial diversity in human feces detected by genusspecific PCR and denaturing gradient gel electrophoresis. Appl Environ Microbiol 67, 504-513.

36. Fuentes S, Egert M, Jimenez-Valera M, et al. (2008) A strain of Lactobacillus plantarum affects segmented filamentous bacteria in the intestine of immunosuppressed mice. FEMS Microbiol Ecol 63, 65-72.

37. Koning CJ, Jonkers DM, Stobberingh EE, et al. (2007) The effect of a multispecies probiotic on the intestinal microbiota and bowel movements in healthy volunteers taking the antibiotic amoxycillin. Am J Gastroenterol 102, 1-12.

38. Hartemink R, Domenech VR \& Rombouts FM (1997) LAMVAB - a new selective medium for the isolation of lactobacilli from faeces. J Microbiol Methods 29, 77-84.

39. Bruinsma N, Hutchinson JM, van den Bogaard AE, et al. (2003) Influence of population density on antibiotic resistance. J Antimicrob Chemother 51, 385-390.
40. Nys S, Okeke IN, Kariuki S, et al. (2004) Antibiotic resistance of faecal Escherichia coli from healthy volunteers from eight developing countries. J Antimicrob Chemother 54, 952-955.

41. De La Cochetiere MF, Durand T, Lepage P, et al. (2005) Resilience of the dominant human fecal microbiota upon short-course antibiotic challenge. $J$ Clin Microbiol 43, $5588-5592$

42. Sullivan A, Edlund C \& Nord CE (2001) Effect of antimicrobial agents on the ecological balance of human microflora. Lancet Infect Dis 1, 101-114.

43. Woodmansey EJ (2007) Intestinal bacteria and ageing. $J$ Appl Microbiol 102, 1178-1186.

44. Goris H, de Boer F \& van der Waaij D (1988) Kinetics of endotoxin release by gram-negative bacteria in the intestinal tract of mice during oral administration of bacitracin and during in vitro growth. Scand J Infect Dis 20, 213-219.

45. Holzheimer RG (2001) Antibiotic induced endotoxin release and clinical sepsis: a review. $J$ Chemother 13, Spec No. 1, $159-172$.

46. Hurley JC (1995) Endotoxemia: methods of detection and clinical correlates. Clin Microbiol Rev 8, 268-292.

47. Hurley JC (1995) Antibiotic-induced release of endotoxin. A therapeutic paradox. Drug Saf 12, 183-195.

48. Ammor MS, Florez AB \& Mayo B (2007) Antibiotic resistance in non-enterococcal lactic acid bacteria and bifidobacteria. Food Microbiol 24, 559-570.

49. Brismar B, Edlund C \& Nord CE (1993) Impact of cefpodoxime proxetil and amoxicillin on the normal oral and intestinal microflora. Eur J Clin Microbiol Infect Dis 12, 714-719.

50. Lode H, Von der Hoh N, Ziege S, et al. (2001) Ecological effects of linezolid versus amoxicillin/clavulanic acid on the normal intestinal microflora. Scand J Infect Dis 33, 899-903.

51. Woodmansey EJ, McMurdo ME, Macfarlane GT, et al. (2004) Comparison of compositions and metabolic activities of fecal microbiotas in young adults and in antibiotic-treated and non-antibiotic-treated elderly subjects. Appl Environ Microbiol 70, 6113-6122.

52. Goossens D, Jonkers D, Russel M, et al. (2003) The effect of Lactobacillus plantarum $299 \mathrm{v}$ on the bacterial composition and metabolic activity in faeces of healthy volunteers: a placebo-controlled study on the onset and duration of effects. Aliment Pharmacol Ther 18, 495-505.

53. Lund B, Edlund C, Barkholt L, et al. (2000) Impact on human intestinal microflora of an Enterococcus faecium probiotic and vancomycin. Scand J Infect Dis 32, 627-632.

54. Tannock GW, Munro K, Harmsen HJ, et al. (2000) Analysis of the fecal microflora of human subjects consuming a probiotic product containing Lactobacillus rhamnosus DR20. Appl Environ Microbiol 66, 2578-2588.

55. Kajander K, Myllyluoma E, Rajilic-Stojanovic M, et al. (2007) Clinical trial: multispecies probiotic supplementation alleviates the symptoms of IBS and stabilises intestinal microbiota. Aliment Pharmacol Ther 27, 48-57. 